\title{
Stromal cells as trend-setters for cells migrating into the lymph node
}

\author{
M Buettner ${ }^{1}$, O Dittrich-Breiholz ${ }^{2}$, CS Falk ${ }^{3}$, M Lochner $^{4}$, A Smoczek $^{5}$, F Menzel $^{1}$, M Bornemann ${ }^{1}$ and \\ U Bode ${ }^{1}$
}

Lymph node stromal cells are known to be immunorelevant during inflammation and tolerance. Differences between peripheral lymph nodes and mesenteric lymph nodes are important for an efficient and effective immune defense. Stromal cells were considered to be perfectly adapted to their draining area and not changeable concerning their expression pattern. Here we show that stromal cells can change their profile after isolation and transplantation into a different draining area. Subsequently, these newly organized lymph nodes are able to induce not only a region-specific but also an antigen-specific immune response. Thus, stromal cells are trend-setters for immune cells in producing a microenvironment that allows an optimized immune defense.

\section{INTRODUCTION}

Lymph nodes (LNs) are located at sites where pathogenic antigens (Ags) might invade the host. They filter the lymph coming from the draining area and initiate immune responses if an $\mathrm{Ag}$ is pathogenic, or tolerance is induced after identification of a harmless Ag. Every LN consists of mobile immune cells, for example, dendritic cells (DCs) and lymphocytes, as well as sessile non-hematopoietic stromal cells (SCs), for example, fibroblastic reticular cells (FRCs) and follicular DCs (FDCs). ${ }^{1,2}$ All these cells are necessary to induce an Ag-specific immune response.

To guarantee an efficient immune reaction for the specific draining area of the LN, differences between the microenvironments of LN are necessary. Focusing on mobile immune cells, gut DCs were found to be positive for $\mathrm{CD} 103^{3}$ and they preferentially expressed the retinal dehydrogenases RALDH2. ${ }^{4}$ Furthermore, DCs from mesenteric LN (mLN) differed from those in peripheral $\mathrm{LN}(\mathrm{pLN})$ in their ability to imprint T cells in the direction of T helper type 2 (Th2), for example, interleukin (IL)- 4 was found at a higher concentration in the mLN compared with the pLN.$^{5-7}$ After activation, T cells and also B cells acquired a gut-homing phenotype, including high CCR9 and $\alpha_{4} \beta_{7}$ integrin expression. ${ }^{8,9}$ Activated $\mathrm{B}$ cells that differentiated into plasma cells in the mLN were found to produce prevalent
IgA, whereas plasma cells induced in pLN were IgG-producing cells. ${ }^{10,11}$

SCs came to the center of attention after their immunological effects were discovered. On high endothelial venules (HEV) of the mLN, MAdCAM-1 was expressed, but not on HEV of pLN. ${ }^{12,13}$ FRCs were found to be RALDH2 producers in mLN, whereas FRC isolated from $\mathrm{PLN}$ produced the B-cell-activating factor at higher levels. ${ }^{13,14}$ Recent studies showed several differences between LN SCs on mRNA level after microarray analysis. ${ }^{13,15}$ All these variations result in an optimal immune reaction to protect the specific draining area of the $\mathrm{LN}$.

The question arises whether the expression profile of SCs is fixed. To answer this point a transplantation model was established. Briefly, pLNs were transplanted into the mesentery and the regenerated LNs were analyzed with respect to cell population and immune response induction. ${ }^{14,16,17}$ Only SCs from the donor were identified in the regenerated LN, whereas immune cells were host-derived. Importantly, these SCs maintained their expression pattern, such as RALDH2 and MAdCAM-1. ${ }^{16,17}$ Thus, SCs sustain their site-specific profile, independent of the draining area after transplantation. Similar results were found transplanting vice versa, showing that MAdCAM-1 expression was retained in the $\mathrm{mLN}$ after transplantation into the skin-draining area. ${ }^{17,18}$ In addition, using a

\footnotetext{
${ }^{1}$ Functional and Applied Anatomy, Hannover Medical School, Hannover, Germany. ${ }^{2}$ Institute of Physiological Chemistry, Hannover Medical School, Hannover, Germany. ${ }^{3}$ Institute of Transplant Immmunology, IFB-Tx, Hannover Medical School, Hannover, Germany. ${ }^{4}$ Institute of Infection Immunology, TWINCORE, Centre for Experimental and Clinical Infection Research (a joint venture between the Medical School Hannover $(\mathrm{MHH})$ and the Helmholtz Centre for Infection Research (HZI)), Hannover, Germany and ${ }^{5}$ Institute of Laboratory Animal Science, Hannover Medical School, Hannover, Germany. Correspondence: M Buettner (Buettner.Manuela@MH-Hannover.de)
} 
single-cell suspension of mLN of newborn mice injected into the skin, further evidence for fixed region-specific LN development was found. Artificial LNs (aLNs) were present showing typical mLN structures attributed to SCs of donorderived origin. ${ }^{19}$ Overall, SCs seem to have a defined expression pattern that is not influenced by the draining area.

A few years ago, Watanabe and colleagues ${ }^{20,21}$ generated an aLN using a thymus-derived SC line (TEL-2-LT $\alpha$ ) combined with bone marrow DCs. These cells were embedded on a collagen matrix and transplanted into the renal subcapsular space of the kidney of mice. After 3 weeks, a LN typical compartment structure with $\mathrm{T}$ - and B-cell area was identified. This aLN was able to induce an Ag-specific immune response. ${ }^{20}$ Germinal centers developed and Ag-specific immunoglobulinproducing plasma cells were detectable. ${ }^{21}$ Furthermore, it was shown that the SC line was the important cell type for LN generation, whereas the DCs only accelerated this process. ${ }^{20}$ However, these SCs were not examined concerning any differences in expression pattern before and after constructing the new LN.

In the present study, we were able to generate a LN using freshly isolated SCs without further in vitro cultivation of these cells. The LN developed, showing lymph and blood vessel connections and normal compartment structures. Furthermore, we isolated SCs from different draining areas to analyze whether region specificity was maintained in these newly generated LN. Within these LN, immune cells can be activated and a region-specific immune response was induced. Thus, SCs, previously shown to have certain established phenotypes, are able to change their expression pattern under isolated conditions.

\section{RESULTS}

\section{Characterization of isolated LN SCs}

LNs are organized in different compartments that are characterized by different non-hematopoietic cell populations. Non-hematopoietic cells (CD45 ${ }^{-}$cells, which are summarized as the SC population) were isolated from $\mathrm{mLN}$ or $\mathrm{pLN}$, and different SC subpopulations were identified using flow cytometry. FRC, lymphatic endothelial cells (ECs), blood ECs, and FDC (gated from the double-negative population) were detected among the $\mathrm{CD}_{4}{ }^{-}$SCs (Figure 1a). The mRNA expression profile of SCs from mLN and pLN was analyzed with microarray analysis. Applying stringent filter criteria to assess differential expression between SCs from mLN and pLN revealed 316 transcripts with elevated mRNA expression in mLN samples and 118 transcripts elevated in pLN (Supplementary Figure 1 online). By focusing on functional groups such as cytokines and chemokines, strong differences in normalized processed signal values could be observed (Supplementary Table 1). This suggested a wide dynamic range of mRNA expression for the different cytokines and chemokines in SCs. Of note, expression levels turned out to be similar between mLN and pLN samples for the majority of analyzed transcripts. In the chemokine group, several genes with high processed signal intensities could be observed. Among these,
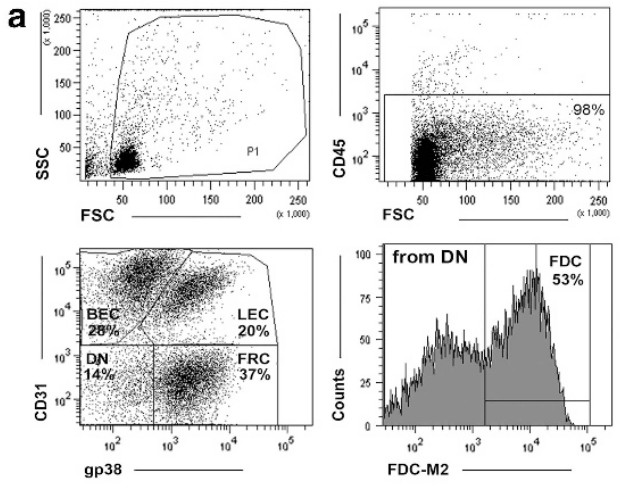

b

\begin{tabular}{|c|c|c|c|c|c|c|c|c|c|}
\hline \multirow[b]{2}{*}{ ProbelD } & \multirow[b]{2}{*}{ Accession } & \multirow[b]{2}{*}{$\begin{array}{c}\text { Gene } \\
\text { symbol }\end{array}$} & \multicolumn{3}{|c|}{ Experiment 1} & \multicolumn{3}{|c|}{ Experiment 2} & \multirow[b]{2}{*}{ Description } \\
\hline & & & $\begin{array}{l}\mathrm{mLN} \\
\text { (nPS) } \\
\end{array}$ & \begin{tabular}{|l|}
$\mathrm{pLN}$ \\
(nPS) \\
\end{tabular} & \begin{tabular}{|c|} 
Fold \\
difference \\
\end{tabular} & $\begin{array}{l}\mathrm{mLN} \\
(\mathrm{nPS}) \\
\end{array}$ & \begin{tabular}{|l|}
$\mathrm{pLN}$ \\
(nPS) \\
\end{tabular} & \begin{tabular}{|c|} 
Fold \\
difference
\end{tabular} & \\
\hline A_51_P464703 & NM_021443 & $\mathrm{CClB}$ & 401 & 1,873 & 4.7 & 322 & 1,812 & 5.6 & chemokine ( $\mathrm{C}-\mathrm{C}$ motif) ligand 8 (CCCB) \\
\hline A_51_P408595 & NM_ 016960 & Co120 & 1,676 & 6,155 & 3.7 & 1,123 & $4: 430$ & 3.9 & chemokine (C-C motif) ligand 20 (C.120) \\
\hline A_51_P209183 & NM_ 019568 & Cxc14 & 2,917 & 349 & -8.4 & 2,117 & 312 & -6.8 & chemokine (CXXC motif) ligand 14 (CXc14) \\
\hline A_51_P384187 & NM_ 011339 & Cxcl15 & 15 & 592 & 39.5 & 15 & 442 & 29.5 & chemokine (CXXC motif) ligand 15 (Cxcl15) \\
\hline$A 51$ P153 & NM 008360 & $\| 18$ & 3,337 & 1,083 & -3.1 & 2.534 & 827 & -3.1 & intereleukin 18 (1118) \\
\hline
\end{tabular}
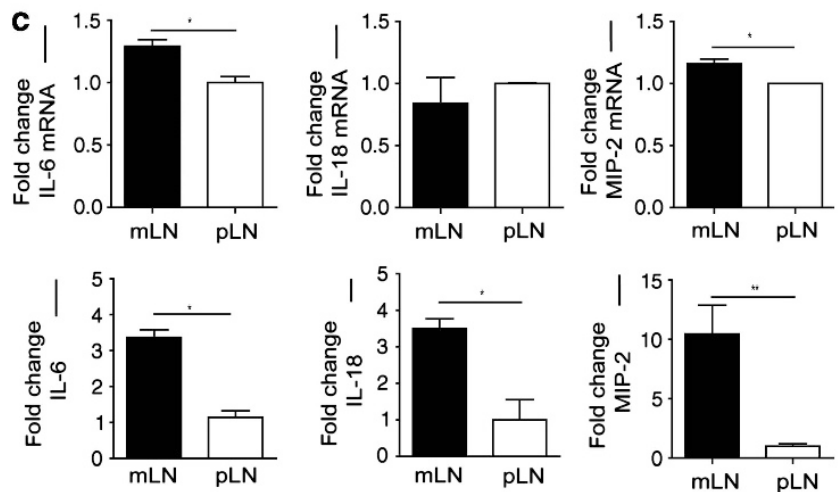

Figure 1 Isolated stromal cells (SCs) from different lymph nodes express different levels of cytokines. CD45 ${ }^{-}$SCs from mesenteric LN $(\mathrm{mLN})$ and peripheral $\mathrm{LN}(\mathrm{pLN})$ were isolated, and flow cytometry, mRNA expression microarray analysis, real-time PCR, and protein detection were performed. (a) Various subpopulations of SCs were identified within CD $45^{-}$cells with a purity of $\sim 98 \%$. Using a combination of anti-CD31 and anti-gp38 antibodies blood endothelial cells (BECs), lymph endothelial cells (LEC), and fibroblastic reticular cells (FRCs) were recognized. Follicular dendritic cells (FDCs) were detected by the anti-FDC-M2 antibody, gated from the CD31/gp38 double-negative (DN) population. (b) Genes encoding interleukins or chemokines that fulfilled the applied filter criteria for different mRNA expressions in $\mathrm{mLN}$ vs. pLN SCs are depicted. Fold difference value ( $\mathrm{pLN} / \mathrm{mLN}$ ) was calculated from normalized processed signal intensities (nPS) and depicted for both independent experiments performed. Agilent Probe-IDs, accession numbers, and gene symbols are given. In cases in which more than one probe directed against the same transcript were present on the microarray and fulfilled the applied filter criteria, one representative probe measurement was selected for visualization. (c) The cytokine and mRNA levels produced by SCs were determined using a bio-plex analysis and real-time PCR, respectively. SCs were isolated and cultured for $24 \mathrm{~h}$. Supernatants and cells were collected and analyzed $(n=3-6)$. Significant differences in the unpaired $t$-test are indicated by ${ }^{*} P<0.05$; ${ }^{* \star} P<0.01$.

CCL19, CCL21 and CXCL13 were identified as the most abundant chemokine transcripts, all of which are well known as chemoattractant molecules for immune cells to migrate into LN. ${ }^{22}$ However, concerning these highly expressed chemokines, no differences were found between $\mathrm{mLN}$ and 
pLN. Only five of the cytokine and chemokine transcripts were consistently found to be differentially expressed more than twofold (Figure 1b; Supplementary Figure 1, Supplementary Table 1). For example, the expression of CCL8 was increased on pLN SCs compared with SCs of the mLN. Very recently, CCL8, also known as monocyte chemoattractant protein 2, was shown to be a ligand for CCR8, which is a mediator for skin-homing. ${ }^{23}$ On the other hand, CXCL14, which is a chemoattractant for monocytes and DC precursors, was detected at higher levels in the mLN SC fraction. ${ }^{24}$ The only differently expressed interleukin was $I L-18$, which is a pro-inflammatory cytokine-stimulating T cells, DCs, and many other cells. ${ }^{25}$ IL-18 was determined in higher amounts in mLN SC. Analyzing the mRNA and protein levels, we also detected higher IL-18 production by SCs of the mLN compared with pLN SCs, whereas IL-6 and MIP-2 production was increased in the supernatants of the mLN SCs (Figure 1c). Thus, SCs from different draining areas showed various differences at the mRNA level but also in protein production. Thereby, SCs have a high impact on establishing the microenvironment of LNs.

\section{Transplanted SCs reorganize a LN}

To investigate the role of SCs and their maintenance of the environment in LN, we isolated SCs from enhanced green fluorescent protein (EGFP) mice, transferred them to a collagen sponge, and transplanted the sponge immediately into two different regions (into the mesentery after removing the mLN or the popliteal region after excising the popliteal LN) in the recipients. Eight weeks after transplantation of SC-loaded collagen sponges, the newly organized LN transplants (Neotx-LN) were excised and analyzed. The connection of the lymph system to the Neotx-LN was clarified by the use of gavaged oil (Figure 2a). Small LNs were found in all transplanted regions (Neotx-LN ${ }^{\text {mes }}$ transplanted in the mesentery or Neotx$\mathrm{LN}^{\mathrm{pop}}$ in the popliteal region), which showed normal compartment structures (Figure 2a). Lymphocytes, as well as DCs or macrophages, repopulated the Neotx-LN. DCs showing a characteristic CD103 expression of the draining area could be identified (Figure 2a and Supplementary Figure 2). The SCs of the Neotx-LN were also analyzed. All subpopulations including FRC, FDC, and lymphatic ECs (Lyve- $1^{+}$cells) were found in the transplants and most of them could be identified as EGFPpositive cells (Figure 2b). However, some EGFP-negative cells were found, which indicates that host-derived SCs were attracted to the newly formed Neotx-LN.

On the other hand, isolated SC subpopulations transplanted into the mesentery resulted in small lymphoid aggregates (when using FRC) or artificial LN structures (when using blood ECs). No lymphoid tissue formation was seen in mock surgeries (sponge transplantation without cells), after lymphatic endothelial cell or after transplantation of the double-negative population (Supplementary Figure 3).
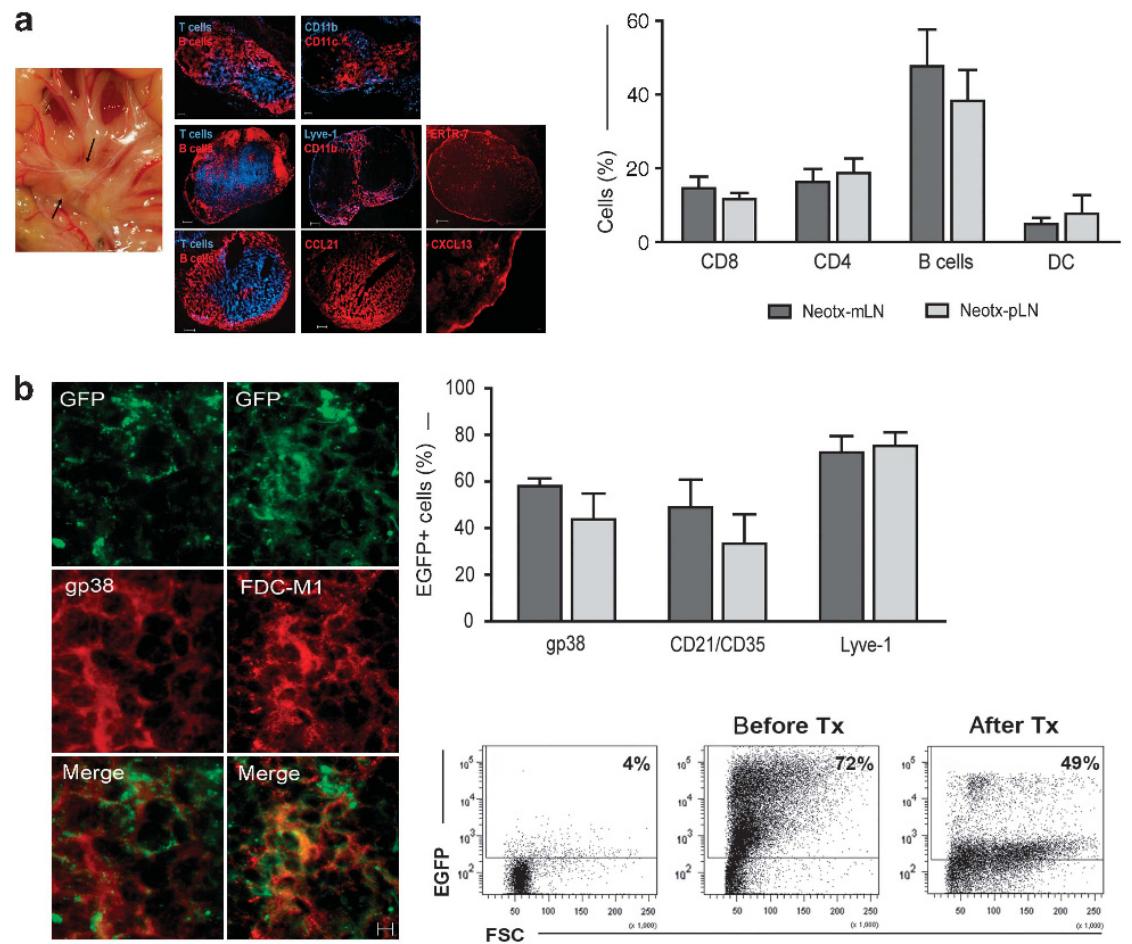

Figure 2 Newly organized lymph nodes develop from transplanted isolated stromal cells (SCs). (a) Eight weeks after transplantation, a lymph node (LN) connected to the afferent lymphatic vessels (indicated by arrows) was generated. Compartments of normal appearance were detected, consisting of Tand B-cell areas but also immigrated dendritic cells (DCs; CD11c) and macrophages (CD11b). LN structures were detected via Lyve-1 or ERTR-7 and also via the presence of area-specific chemokines such as CCL21 in the T-cell area or CXCL13 in the B cells. Flow cytometry analysis showed similar percentages of all immune cells, independent of whether SCs from mesenteric $L N(m L N)$ or peripheral $L N(p L N)$ were transplanted $(n=8)$. (b) Using immunofluorescence histochemistry or flow cytometry, EGFP ${ }^{+}$cells were identified predominantly in the group of SCs. EGFP ${ }^{+}$fibroblastic reticular cell (FRC) $\left(\mathrm{gp} 8^{+}\right)$, follicular DC (FDC; CD21/35+ ) but also Lyve-1 ${ }^{+}$cells were found in similar percentages in the newly generated LN. The means and s.e. are given from three to six independent experiments. EGFP, enhanced green fluorescent protein. 



Figure 3 MAdCAM-1 is induced on donor-derived endothelial cells. EGFP ${ }^{+}$stromal cells (SCs) isolated from peripheral LN (pLN) and mesenteric LN $(\mathrm{mLN})$ were transplanted into the mesentery (mes) and popliteal fossa (pop), respectively. Eight weeks later, the newly generated lymph node (LN) were removed and cryosections of the transplanted LN fragments (Neotx-LN) or control LNs were stained for high endothelial venule (HEV; anti-CD31, blue) and MAdCAM-1 (red). HEV s of control mLN were found to be double-positive, similar to HEV in Neotx-pLN ${ }^{\text {mes }}$, whereas control pLN and Neotx-mLN ${ }^{\text {pop }}$ showed no MAdCAM-1 staining on HEV. EGFP, enhanced green fluorescent protein.

Thus, transplanted SCs alone were able to reconstruct a LN that showed normal compartment structures and immune cell subsets after regeneration.

\section{MAdCAM- 1 is expressed in Neotx-pLN transplanted into the mesentery}

MAdCAM-1 is a gut-specific molecule located on EC and is exclusively expressed by HEV in the mLN but not in other LNs (Figure 3) ${ }^{12}$ Using EGFP mice, the origin of HEV in Neotx-LN was clarified. To identify HEV in Neotx-mLN ${ }^{\text {pop }}$ and Neotx$\mathrm{pLN}^{\mathrm{mes}}$, Neotx-LNs were stained immunohistologically with anti-CD31 and anti-MAdCAM-1 antibodies. All Neotx-LNs developed HEV, identified by $\mathrm{CD} 31$, which were found to be also EGFP ${ }^{+}$. Thus, the transplanted ECs were able to reorganize the vascularization of the Neotx-LN. Furthermore, we were interested in the expression of MAdCAM-1 in Neotx-LN. MAdCAM-1 was found on HEV within both transplanted Neotx-LN ${ }^{\text {mes }}$, but not on HEV in Neotx-LN ${ }^{\text {pop }}$ (Figure 3). These findings suggest that the draining area has a role in the vascularization of the Neotx-LN. MAdCAM-1 seems to be inducible on ECs through stimulation by unknown factors coming from outside the LN. Thus, the draining area has an effect on single ECs during the reorganization of vascularization.

\section{Donor-derived SCs change their characteristic behavior after transplantation}

After transplantation of LN, SCs were shown to retain their characteristic expression of several molecules such as
RALDH2 or MAdCAM- $1 .{ }^{16}$ As illustrated above, MAdCAM-1 expression changed after SC transplantation. To identify whether these changes were induced by host- or donor-derived SCs, the expression of characteristic mRNA for mLN or pLN SCs was analyzed. Host- and donor-derived SCs (EGFP - or EGFP + ) from Neotx-mLN ${ }^{\text {mes }}$ and Neotx-pLN ${ }^{\text {mes }}$ were isolated, sorted (Supplementary Figure 4), and analyzed for mRNA expression of RALDH2, as an indication for guthoming induction, as well as $I L-18$ and CXCL14, as genes that differ in expression between LN. As already known, RALDH2 expression on pLN SCs was found to be decreased compared with mLN SCs. However, after transplanting pLN SCs into the mesentery, host-derived SCs (EGFP - cells) from Neotx$\mathrm{pLN}^{\text {mes }}$ showed an expression profile of an mLN. Surprisingly, in donor-derived pLN SCs (EGFP + cells) an expression of RALDH2 was detectable (Figure 4). In addition, the mRNA expression levels for $I L-18$ and CXCL14 were analyzed. $I L-18$ expression was detectable in all Neotx- $\mathrm{mLN}^{\text {mes }}$ fractions and also in the EGFP - and EGFP + Neotx-pLN ${ }^{\text {mes }}$ SCs (Figure 4). Furthermore, the expression of CXCL14 was highly upregulated in pLN SCs after transplantation into the mesentery. Thus, SCs, which had previously been shown to maintain their site-specific expression pattern, changed their mRNA profile after isolation from the cell-cell contact. During reorganization of a new united cell structure and regeneration of $\mathrm{LN}$, SCs seem to be able to change to regionspecific SCs. 

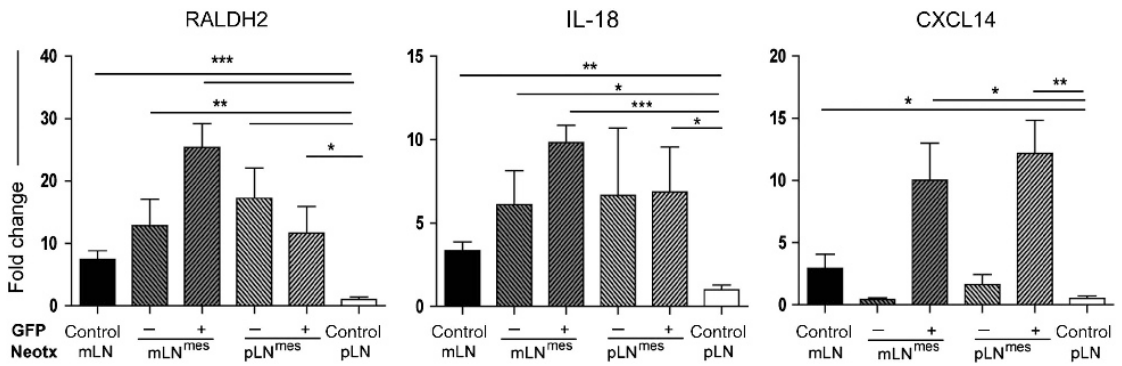

Figure 4 Stromal cells (SCs) from peripheral LN (pLN) change their expression pattern to that of mesenteric $L N(m L N)$. Neotx-mLNmes and Neotx$\mathrm{pLN}^{\text {mes }}$ were removed 8 weeks after transplantation and SCs were sorted into an EGFP ${ }^{+}$and EGFP ${ }^{-}$fraction using a FACSAria llu. $\mathrm{mRNA}$ was isolated and real-time PCR in triplets was performed. All SCs isolated from Neotx-LN ${ }^{\text {mes }}$ showed a similar expression of genes compared with the mLN control. The data are normalized to the housekeeping gene $\beta$-actin and given from four animals. Significant differences in the unpaired $t$-test are indicated by ${ }^{\star} P<0.05 ;{ }^{* \star} P<0.01 ;{ }^{* \star \star} P<0.001$.

\section{Neotx-LN SCs transplanted into the mesentery support the generation of gut-homing $T$ cells}

To investigate the type of tissue tropism generated in Neotx-mLN or pLN, T-cell receptor (TCR) transgenic OT-II T cells were fluorescently labeled with 5-(and 6)-Carboxyfluorescein diacetate succinimidyl ester and adoptively transferred into nonmanipulated or transplanted recipients. The presence of adoptively transferred cells in Neotx-LN indicated that Neotx-LNs were vascularized to the blood system as well as the lymph system. One day later, Neotx- $\mathrm{LN}^{\text {mes }}$ mice were fed with ovalbumin (Ova), and Neotx-LN ${ }^{\text {pop }}$ mice received a single subcutaneous injection. Three days after Ag delivery, Neotx-LN from both transplanted sites (mesentery and popliteal region) were isolated and analyzed with flow cytometry. In line with previous reports, we observed that in nontransplanted wild-type (wt) mice, Ova administration induced T-cell proliferation in the gut-draining $\mathrm{mLN}$ as well as pLN (Figure 5a); however, a gut-homing phenotype, characterized by upregulation of $\alpha_{4} \beta_{7}$ integrin and CCR9, was acquired only in the mLN (Figure 5b). OT-II cells were found in all NeotxLN in similar percentages, and after Ova administration proliferation of these cells was measured within the Neotx$\mathrm{LN}$ (Figure 5a). Staining of CCR 9 and $\alpha_{4} \beta_{7}$ integrin on OT-II cells was detectable also in equal percentages comparing control mLN and Neotx-pLN ${ }^{\text {mes }}$, whereas in Neotx-mLN ${ }^{\text {pop }}$ upregulation was not found (Figure $\mathbf{5 b}$ ). To eliminate the possibility of OT-II cell priming in areas other than as the Neotx-LN, we transplanted wt SC into ROR $\gamma$ t mice $\left(\operatorname{Neotx}^{\star}-\mathrm{pLN}^{\text {mes }}\right)$, which do not have innate LN. Likewise, these $\mathrm{Neotx}^{\star}-\mathrm{pLN}^{\text {mes }}$ were smaller than LN transplanted into wt mice, OT-II cell proliferation was detectable, and CCR9 and $\alpha_{4} \beta_{7}$ integrin upregulation were measured in similar percentages as previously analyzed (Figure 5b). Thus, cells that migrated into the newly developed LN were activated and showed gut-homing properties after $\mathrm{Ag}$ recognition. Together, these data demonstrate that SCs from pLN, which normally do not induce gut-homing, changed their profile after transplantation and were able to direct lymphocytes to a gut-specific phenotype.

\section{Neotx-pLN ${ }^{\text {mes }}$ are able to induce a cholera toxin-specific immune response}

Although gut-homing molecules were shown to be induced on OT-II cells after Ag administration in Neotx-pLN ${ }^{\text {mes }}$, it was not
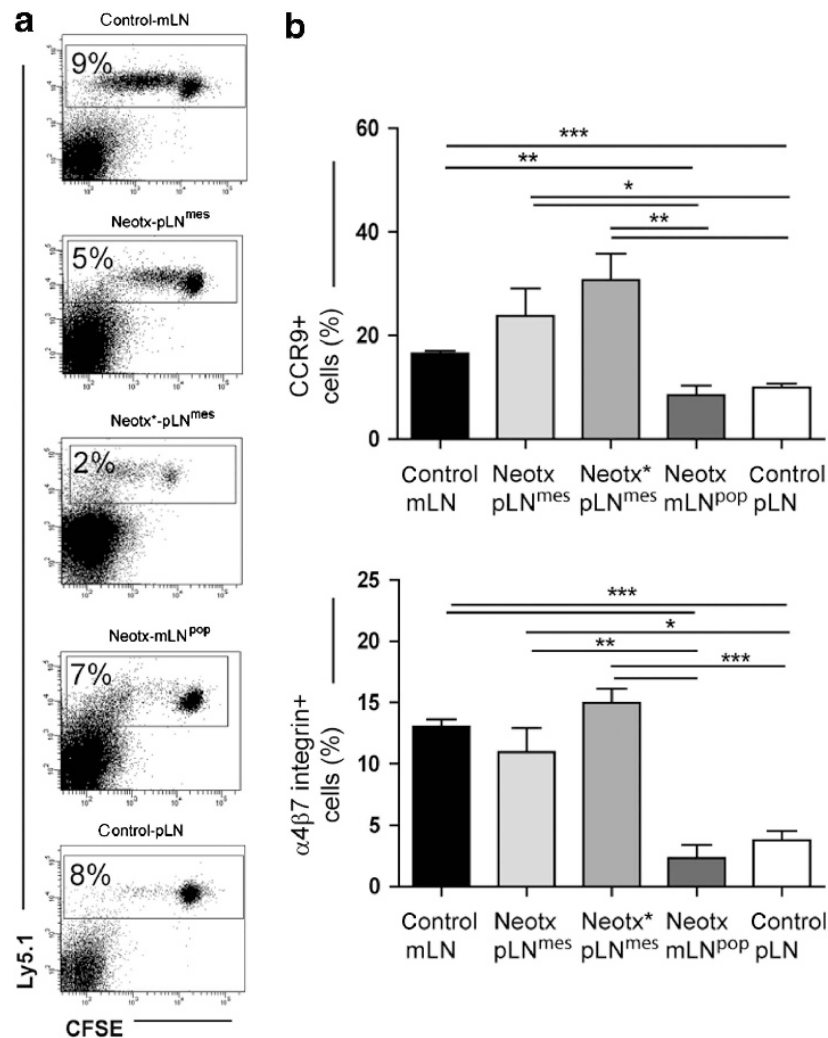

Figure 5 Transferred antigen-specific lymphocytes proliferate and induce gut-homing molecules in Neotx from isolated peripheral LN (pLN) stromal cells (SCs). Eight weeks after transplantation, CFSE, 5-(and 6)Carboxyfluorescein diacetate succinimidyl ester-labeled spleen cells isolated from OT-II mice were adoptively transferred into transplanted (wild-type recipients (Neotx-LN ${ }^{\text {mes }}$ ) or ROR $\gamma$ t recipients $\left(\right.$ Neotx $\left.^{*}-\mathrm{pLN}^{\mathrm{mes}}\right)$ ) and non-transplanted control recipients. One day after transfer, mice received a single administration of ovalbumin (Ova), and after 3 days lymph nodes (LNs) were excised and CCR9 and $\alpha_{4} \beta_{7}$ integrin upregulation was determined with flow cytometry. OT-Il cells were found in all lymph node (LN) in similar percentages (a). Neotx-LN ${ }^{\text {mes }}$ induced gut-homing molecules independent of the origin of SCs, which generated the LN, whereas no upregulation was identified in Neotx-LN ${ }^{\text {pop }}$ (b). The means and s.e. are given from four to eight independent experiments (significant differences in the unpaired $t$-test are indicated by ${ }^{\star} P<0.05$; ${ }^{\star *} P<0.01$; $\left.{ }^{\star \star \star} P<0.001\right)$.

clear whether the Neotx-pLN ${ }^{\text {mes }}$ could induce an Ag-specific immune response including Ag-specific IgA production. Therefore, we fed cholera toxin (CT) to control and 

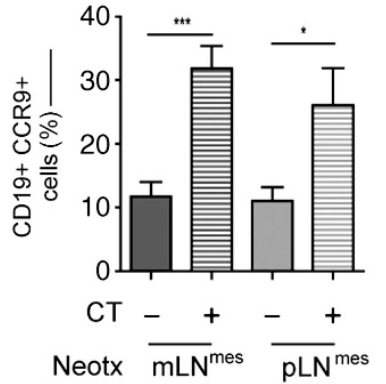

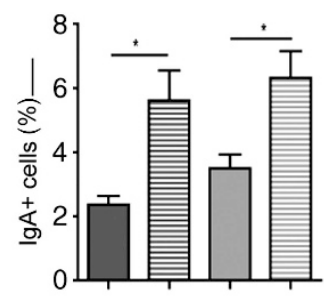

CT $\frac{-}{\operatorname{mLN}^{\text {mes }}} \frac{-}{\mathrm{pLN}^{\text {mes }}}$

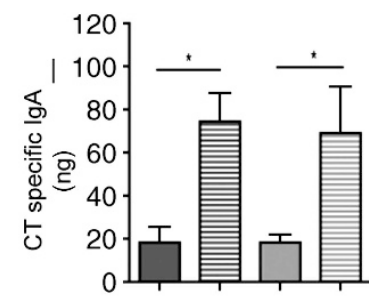

CT $\frac{-}{\mathrm{CL}_{\mathrm{mLN}}}{ }^{\mathrm{mes}} \frac{-}{\mathrm{pLN}^{\mathrm{mes}}}$

Figure 6 Cholera toxin-specific immune response is initiated in newly generated lymph node (LN) independent of the stromal cell (SC) origin. It was analyzed whether the Neotx-pLN ${ }^{\text {mes }}$ is able to induce a specific immune response, which includes the induction of IgA-producing cells. Cholera toxin (CT) was given three times and, after 19 days, LNs were removed and the B-cell phenotype was analyzed using flow cytometry. The percentage of surface $\mathrm{IgA}^{+} \mathrm{B}$ cells was similar in Neotx-pLN ${ }^{\text {mes }}$ compared with Neotx-mLN ${ }^{\text {mes }}$ after CT administration. CCR9 expression was upregulated on B cells from Neotx-pLN ${ }^{\text {mes }}$, and thus similar percentages were found compared with Neotx-mLN ${ }^{\text {mes }}$. Furthermore, the gut was lavaged to perform a CT-specific IgA enzyme-linked immunosorbent assay. Data show comparable CT-specific lgA levels in Neotx-mLN ${ }^{\text {mes }}$ and Neotx-pLN ${ }^{\text {mes }}$. This indicates that gut-specific $B$ cells were induced in Neotx-pLN ${ }^{\text {mes }}$ after CT treatment. The means and s.e. are given from three to eight independent experiments (significant differences in the unpaired $t$-test are indicated by ${ }^{\star} P<0.05$; ${ }^{* \star *} P<0.001$ ).

Neotx-LN ${ }^{\text {mes }}$ mice 8 weeks after transplantation and analyzed the Neotx-LN $\mathrm{LN}^{\text {mes }}$ for IgA-producing cells, the induction of guthoming molecules as well as CT-specific IgA in the intestinal lavage. Independent of the origin of SCs, we found similar percentages of IgA-producing cells and B cells, which had upregulated CCR9 for an efficient homing into the gut in the Neotx- $\mathrm{LN}^{\mathrm{mes}}$ (Figure 6). In the intestinal lavage, the CT-specific IgA production was found to be upregulated in Neotx- $\mathrm{LN}^{\text {mes }}$ to the same extent after $\mathrm{CT}$ administration. Thus, the Neotx-pLN ${ }^{\text {mes }}$ were able to induce and activate lymphocytes in the direction needed in the draining area.

\section{Regeneration of LNs via proliferation and reprograming}

Neotx- $\mathrm{LN}^{\text {mes }}$ were analyzed one, two, and four days after transplantation to identify how transplanted SCs are involved in the process of LN organization. At all time points, we found SCs that were gp $38^{+}, \mathrm{FDC}-\mathrm{M} 2{ }^{+}, \mathrm{CD} 31^{+}$, or Lyve- $1^{+}$and immune cells positive for $\mathrm{CD} 3$ and B220. The immune cells were found to be $\mathrm{GFP}^{-}$and they formed small T- and B-cell clusters already 2 days after transplantation (Supplementary Figure 5). As expected, the $\mathrm{GFP}^{+}$cell population was positive for gp38, CD31, FDC-M2, and Lyve-1 (Supplementary Figure 5); however, we also found cells that were positive for Dlk-1 (Figure 7). Dlk-1, also known as Pref-1, is a marker for adipocyte and mesenchymal progenitor cells. ${ }^{26}$ These cells were previously shown to be able to differentiate into SCs of $\mathrm{LN}^{27}$ In control, $\mathrm{mLN}$ Dlk-1 ${ }^{+}$cells were also detectable but were found to be negative for further markers such as CD45, CD11c, CD31, and FDC-M2 (Supplementary Figure 6). A double staining with Dlk-1 and gp38 showed a heterogeneous picture. We identified gp $38^{-}$Dlk-1 ${ }^{+}$cells as well as gp $38^{+}$ and Dlk-1 ${ }^{+}$cells (Supplementary Figure 6). One day after transplantation, only few Dlk- ${ }^{+} \mathrm{GFP}^{+}$cells were detectable; however, on day two we identified small clusters of these cells (Figure 7). In addition, many $\mathrm{Ki}^{+}{ }^{+}$cells were found at that time point (Figure 7). Ki67 was used as a marker for proliferation and $\mathrm{Ki}_{67}{ }^{+}$cells were stained in combination
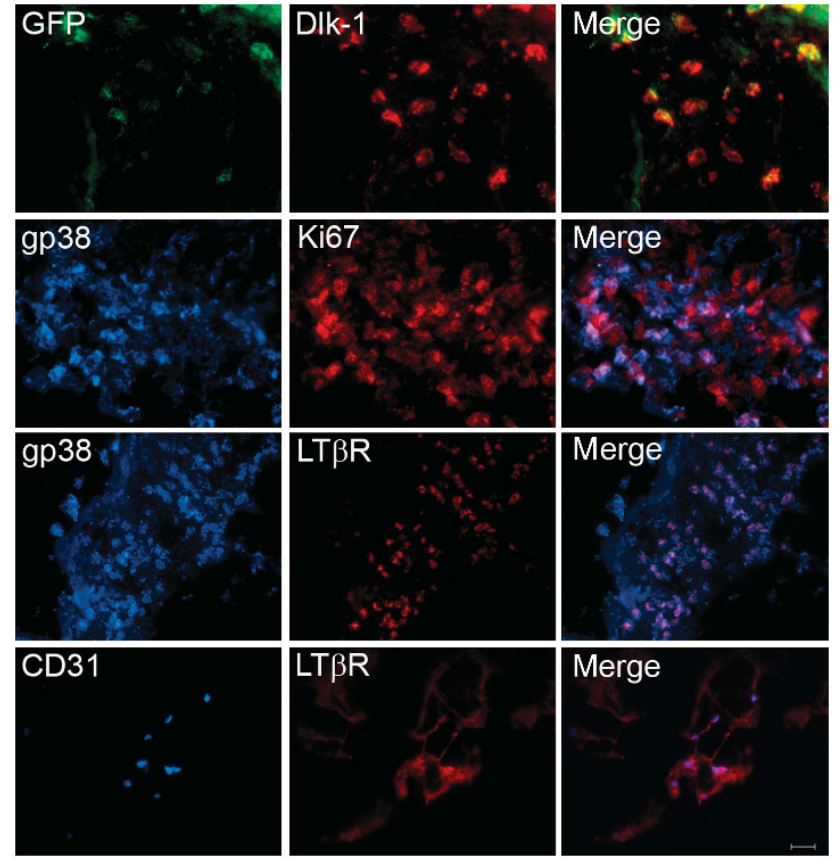

Figure 7 Two days after transplantation, stromal cells (SCs) initiated reorganization. Isolated $\mathrm{EGFP}^{+} \mathrm{SC}$ s were embedded on a collagen sponge and transplanted into the mesentery of wild-type (wt) mice. Two days after transplantation, Neotx-LN (lymph node) was excised and analyzed for mesenchymal stem cells (anti-Dlk-1), proliferation (antiKi67), and LT $\beta$ R expression. Numerous Dlk- $1^{+}$EGFP ${ }^{+}$cells are seen in the border of Neotx-LN, as well as proliferating cells $\left(\mathrm{Ki}_{6} 7^{+}\right)$, which are mostly gp38 ${ }^{+}$, and LT $\beta R^{+}$cells, which were also found to be gp38 or CD31-positive $(n=4)$. Bar $=20 \mu \mathrm{m}$. EGFP, enhanced green fluorescent protein.

with gp38 as an example of transplanted SCs. Double-positive cells were observed already 2 days after transplantation, which indicated a high proliferation capacity. Furthermore, we found $\mathrm{LT} \mathrm{R}^{+}$cells that were also positive for gp38 or CD31 (Figure 7). These cells were also found in higher numbers 
2 days after transplantation. Analyzing the mRNA expression of Neotx-LN ${ }^{\text {mes }}$, we also observed a slightly upregulated expression of LT $\beta R$ as well as LT $\alpha$ and CXCL13 on day 1 compared with day 4 after transplantation (Supplementary Figure 7). All these molecules are known to be involved in LN development; however, especially LT $\beta$ R was found to be expressed on ${\mathrm{gp} 38^{+}}^{+}$cells as well as $\mathrm{CD} 31^{+}$cells. $^{28}$ Thus, transplanted mesenchymal progenitor cells (Dlk-1 + cells) seem to be involved in the organization of LN but also transplanted SCs positive for gp38 or CD31 may have an impact. On one hand, these cells proliferated and, on the other hand, they upregulated proteins that are important during LN development.

\section{DISCUSSION}

It is generally accepted that SCs of LN are determined in their expression pattern after differentiation. In the recent past, differences in immunorelevant genes were detected between SCs isolated from the peripheral site and those from the mesenteric site. ${ }^{12-15}$ This is clearly connected to different homing characteristics of immune cells into the skin or into the gut. ${ }^{82,29}$ Our study documented for the first time the transformation of immunorelevant molecules expressed by SCs isolated from $\mathrm{mLN}$ or pLN. Consequently, this leads to a shift in the homing appearance of lymphocytes in the draining area.

A well-established hypothesis is that the skin-draining LNs (pLN) and gut-draining LN (mLN), which drain different areas of the body, show differences concerning immune cell activation and their microenvironment. The backbone forming SCs of LN were found to be involved in the development of the LN-specific environment. ${ }^{14,16,30,31}$ MAdCAM-1 is found on HEV of mLN but not on HEV of pLN. ${ }^{12}$ Isolated SCs of mLN and pLN showed differences in the expression of RALDH2, which was reported to be involved in the induction of guthoming molecules on lymphocytes. ${ }^{14,16}$ Microarray analyses performed on SCs isolated from different draining areas to identify further differences between the $\mathrm{LN}^{13,15}$ found differences in cell numbers, extracellular matrix components, and cell-cell interaction molecules. ${ }^{13}$ Cytokine levels were also analyzed. ${ }^{15}$ Our results illustrated that chemokines such as CCL19 and CCL21 are highly expressed in both LN. These are important chemokines for cell migration into the LN for lymphocytes as well as DC. ${ }^{32}$ In mice deficient for these chemokines, the migration was disturbed, normal compartment structures were absent, and immune response as well as tolerance induction were impaired. ${ }^{33,34}$ In addition, our results showed further differences between isolated SCs and SC subpopulations from different regions with respect to mRNA expression pattern, but more importantly also with respect to protein levels. IL-18, which has been shown to enhance T-cell activation and IL-4 production to a typical Th2 response, ${ }^{25}$ was found to be prevalently produced in mLN SCs. This is in line with previous findings where IL- 4 was predominantly found in mLN compared with pLN. ${ }^{5}$ Furthermore, IL-18 was shown to have direct effects, not only on DCs and macrophages but also on fibroblast and ECs that upregulate vascular cell adhesion molecule 1 and intercellular adhesion molecule $1 .^{25}$ The mRNA level of IL-6 was found to be decreased on mLN SCs compared with $\mathrm{pLN} ;{ }^{13}$ however, analyzing the supernatants of a $24-\mathrm{h} \mathrm{SC}$ culture IL-6 was detected at higher levels in mLN compared with pLN culture. This shows that protein level detection is an additional tool for microenvironment analysis. IL-6 is a cytokine, which also drives an immune response in the Th2 direction. ${ }^{35}$ These findings suggest a high impact of SCs on immune cell activation and on the direction of the immune response.

Differences were found for homing molecules on activated lymphocytes such as CCR9 and $\alpha_{4} \beta_{7}$ integrin in mLN and CCR8 and CCR4 in pLN. ${ }^{8,12,23}$ Th2 cells were detected more frequently in $\mathrm{mLN}$ that produce the cytokines IL-4 and transforming growth factor $\beta{ }^{5}$ In contrast, pLN lymphocytes were primed to the Th1 site secreting IL-2 and interferon $\gamma^{5}{ }^{5}$ From the lamina propria of the gut, $\mathrm{MHCII}^{+} \mathrm{CD} 03^{+} \mathrm{DCs}$ were exclusively shown to migrate to the $\mathrm{mLN}^{29}$

This beneficial connection between SCs and lymphocytes was identified using a transplantation model. ${ }^{14,16,18,36}$ Whole LNs from different draining areas were transplanted and well-known differences were analyzed after regeneration. Only SCs of the transplants were found to be host-derived, whereas only immune cells from the donor were detectable. ${ }^{14,16}$ On SCs of pLN transplanted into the mesentery, MAdCAM-1 and RALDH2 were not inducible, whereby lymphocytes were not able to upregulate CCR9 and $\alpha_{4} \beta_{7}$ integrin. ${ }^{16}$ Consequently, an Ag-specific immune response was not induced after transplantation of the pLN into the mesentery. ${ }^{16}$ In conclusion, the remaining SCs of these pLNtx were trend-setters for all cells migrating into the $\mathrm{LN}$.

In various studies, SCs of LN were shown to be connected to each other within their organ and after transplantation a similar connection is most likely. An interesting question is whether SCs need this association to form a LN and to maintain their specific expression pattern. In this study, isolated $\mathrm{EGFP}^{+}$SCs from $\mathrm{pLN}$ and $\mathrm{mLN}$ were transplanted, and fully regenerated LNs were found, which showed normal compartment structures and were connected to the lymph as well as to the blood system. However, the recovery of $\mathrm{EGFP}^{+}$cells after new LN formation indicates that host-derived SCs were recruited from the periphery. Previously, the generation of an aLN from cultured SCs (TEL-2-LT $\alpha$ ) was achieved. ${ }^{20,21}$ TEL-2-LT $\alpha$ cells with or without DCs were transplanted and the potential of these cells to generate functional LN was analyzed. The transplants were removed and TEL-2-LT $\alpha$ cells identified as the significant cell type for the generation of LN, with DCs as a supporting cell population. ${ }^{20}$ However, the expression profile of these SCs after creating a new LN was not analyzed. The present study focuses on the SCs and their expression pattern after creating a new LN.

After transplantation of whole $\mathrm{mLN}$ into the cervical region, HEVs of mLN maintain their expression of MAdCAM- $1 .{ }^{18}$ On HEV s of pLN, which do not show MAdCAM-1 expression, this was not inducible during regeneration in the mesentery. ${ }^{16}$ However, after transplantation of isolated $\mathrm{EGFP}^{+}$pLN SCs 
into the mesentery, MAdCAM-1 was found on HEV on donorderived ECs. Vice versa, after transplantation of MAdCAM-1 ${ }^{+}$ $\mathrm{EGFP}^{+}$mLN SCs into the popliteal fossa, MAdCAM-1 ${ }^{+}$ HEVs were not found. Impressively, MAdCAM-1 is changeable after re-construction of a new LN by isolated SCs. Cupedo et al. ${ }^{19}$ injected intradermally a single-cell suspension of all mLN cells into the skin of mice where LN generation was nonpredestined. After regeneration of a LN structure they found MAdCAM-1 expression on HEV. They described functional blood vessels but an inefficient connection of the LN structure to the lymph vessel system. ${ }^{19}$ This difference to our finding of MAdCAM-1 expression seems to be dependent on an optimal contact of LN and the lymphatic system.

Furthermore, in the present study RALDH2, CXCL14, and IL-18 expression levels were found to be induced in pLN SCs after transplantation into the mesentery. Thus, pLN SCs induce gene expression of molecules normally found on mLN SCs. The draining areas, which seem to be of no importance for SC expression after whole LN transplantation, showed a high impact on newly formed LNs.

RALDH2 was found to be an important enzyme in the induction of gut-homing on immune cells. ${ }^{4}$ Neotx-pLN ${ }^{\text {mes }}$ SCs upregulated the expression of RALDH2; therefore, next the potential of the newly generated LN for the induction of CCR9 and $\alpha_{4} \beta_{7}$ integrin was analyzed. Both were found to be induced on injected T cells in Neotx- $\mathrm{LN}^{\text {mes }}$ similar to cells identified in control mLN. The same experiment was performed in mice with whole LN transplants. Ag-specific T cells in pLNtx showed reduced induction of these molecules compared with mLNtx. ${ }^{14}$ Thus, SCs change their expression pattern under isolated conditions, contrary to former conceptions. These changes resulted in a different homing profile of $\mathrm{T}$ cells.

Similar to T-cell activation, the activation of B cells for antibody production is also important for an efficient immune response. B cells of transplanted pLN were not able to induce CCR9 and showed no class switch to IgA after Ag administration. ${ }^{16}$ This was not true for Neotx-pLN ${ }^{\text {mes }}$ where lymphocytes were found to be gut-specific. This result confirms the hypothesis that isolated SCs transplanted into a different draining area change their site-specific profile and react subsequently as a draining area-specific LN. Finally, LN SCs are not strongly restricted in their expression pattern but can change their profile after isolation and reorganization.

Reorganization of lymphoid tissues was found after viral infection. ${ }^{37-39}$ During this process, pathways that were activated during LN development seem to be reactivated. For reorganization of the SC network, the cross-talk between gp $38^{+}$SCs and lymphoid tissue inducer cells was found to be dependent on IL-7 and LT $\beta$ R. ${ }^{38,39}$ Two days after transplantation of SCs, we saw high numbers of $\mathrm{LT} \mathrm{R}^{+}$, gp $38^{+}$cells but also $\mathrm{LT} \mathrm{R}^{+} \mathrm{CD} 31^{+}$cells. LT $\beta \mathrm{R}$ on ECs was shown to be important in the formation of LN and also in HEV development. Without LT $\beta R$ on EC, the expression of CCL21 and CCL19 was reduced and the migration of lymphocytes was impaired. ${ }^{28}$ The findings of this study support the assumption that isolated and transplanted SCs re-expressed molecules such as LT $\beta R$, which are important and functional during LN development. Furthermore, Dlk- ${ }^{+}$cells were detected within the SC population before and after transplantation. Cells positive for this marker were identified as mesenchymal stem cells or adipocyte progenitor cells. ${ }^{26}$ These cells were found to differentiate into LN SCs via LT $\beta R$ stimulation. ${ }^{27}$ Taken together, these results suggest that LN SCs reorganize a new LN via re-differentiation to $\mathrm{LN}$ organizer cells. Afterwards, these cells not only seem to activate differentiation of stem cells but also proliferate and attract further cells to the area. Acting in concert, transplanted SCs and mesenchymal stem cells arrange a new LN structure that is optimally adapted to the draining area.

In conclusion, this study demonstrates for the first time that SCs are changeable in their expression pattern and thereby the environment of the LN can change to be site-specific. These changes allow the induction of homing molecules on lymphocytes and the class switch to IgA, which is important for a normal gut homeostasis or immune reaction. SCs of LN are a key cell population for an efficient and specific immune system.

\section{METHODS}

Animals. Female C57BL/6, C57BL/6-Tg (ACTbEGFP; designated here as EGFP mice), C57BL/6.129P2(Cg)-Rorc ${ }^{\text {tm2Litt } / J ~(d e s i g n a t e d ~ h e r e ~ a s ~}$ ROR $\gamma \mathrm{t}$ mice), and C57BL/6-Tg(Tcra Tcrb)425Cbn-Ptprca (designated here as OT-II mice; T cells of these mice selectively recognize Ova peptide presented in the context of MHC class II) mice were bred at the central animal laboratory of Hannover Medical School and were used at a weight of $18-25 \mathrm{~g}$. All animal experiments were performed in accordance with the institutional guidelines and had been approved by the Niedersächsisches Landesamt für Verbraucherschutz und Lebensmittelsicherheit (No. 33.9-42502-04-09/1667).

SC isolation. For isolation of CD $45^{-}$SCs, the $\mathrm{mLN}$ or the pLN of EGFP or wt mice were removed and $\mathrm{LN}$ were digested at $37^{\circ} \mathrm{C}$ for $30 \mathrm{~min}$ with $1 \mathrm{mg} \mathrm{ml}^{-1}$ Collagenase 8 (Sigma-Aldrich, St Louis, MO) in RPMI $1640 / 10 \%$ fetal calf serum. CD $45^{-}$cells were isolated using the MACS technique following the instructions provided by Miltenyi (BergischGladbach, Germany). The mean value of purity of $\mathrm{CD} 45^{-}$cells was $97.4 \% \pm 2.0$ for $\mathrm{mLN}$ cells and $97.5 \% \pm 2.2$ for pLN. The SCs were used for mRNA isolation or embedded on a collagen sponge (CS-35; Koken, Tokyo, Japan) and transplanted into donor mice.

Microarray analysis. In two independent experiments, $\mathrm{CD} 45^{-}$cells from pLN and mLN were isolated and total RNA was isolated according to the manufacturer's protocol (RNeasy Micro Kit, Qiagen, Hilden, Germany). Samples from all individuals of the same genotype were pooled. The Whole Mouse Genome Oligo Microarray $4 \times 44 \mathrm{~K}$ (G4122F, design ID 014868, Agilent Technologies, Santa Clara, CA) was employed in this study. In all, $80 \mathrm{ng}$ of total RNA was used to prepare Cy3-labeled cRNA (Amino Allyl MessageAmp II Kit; Life Technologies, Karlsruhe, Germany) as recommended by the company. cRNA fragmentation, hybridization, and washing steps were carried out according to Agilent's One-Color Microarray-Based Gene Expression Analysis Protocol V5.7, except that $400 \mathrm{ng}$ of each labeled cRNA sample was used for hybridization. Slides were scanned on the Agilent Micro Array Scanner G2505 B at two different PMT settings (100 and 5\%) to increase the dynamic range of the measurements. Data extraction was performed with the "Feature Extraction Software V9.5.3.1" by using the recommended default extraction protocol file: GE1-v5_95_Feb07.xml. Processed intensity values ("ProcessedSignal" or "PS") were globally normalized by a linear scaling approach. All PS 
values of one sample were multiplied by an array-specific scaling factor. This scaling factor was calculated by dividing a "reference 75 th Percentile value" (set as 1,500 for the whole series) by the 75th Percentile value of the particular Microarray ("Array i" in the formula shown below). Accordingly, normalized PS values for all samples (microarray data sets) were calculated by the following formula:

$$
\text { Normalized } \mathrm{PS}_{\text {Array } \mathrm{i}}=\mathrm{PS}_{\text {Array } \mathrm{i}} \times\left(1,500 / 75^{\text {th }} \text { Percentile }_{\text {Array } \mathrm{i}}\right)
$$

A lower intensity threshold was defined as $1 \%$ of the reference 75 th Percentile value $(=15)$. All normalized PS values that fell below this intensity border were substituted by the respective surrogate value of 15. Normalized PS values of on-chip replicate probes were averaged arithmetically before ratio calculation.

Whole data were filtered for transcripts that fulfilled the following criteria: (1) fold difference in processed signal intensities calculated from each possible pair of $\mathrm{mLN}$ vs. pLN samples more than twofold (consistent and unidirectional); (2) the arithmetic mean of processed signal intensities calculated from each possible pair of mLN vs. pLN samples >100; (3) technical impairment, as defined by the entry "IsFeatNonUnifOL" (Feature extraction software) absent $(=0)$ in each of the four samples analyzed.

Intestinal surgery. $\mathrm{CD} 45^{-} \mathrm{SCs}$ from $\mathrm{mLN}$ and $\mathrm{pLN}$ were isolated from C57BL/6 or EGFP mice and used as donors for C57BL/6 mice and ROR $\gamma$ t mice. Under the combined anesthesia with Ketamine (Gräub AG, Bern, Switzerland) and xylazine 2\% (Bayer Health Care, Leverkusen, Germany), the mLNs of the small and large intestine or the popliteal LN of the host were excised and donor SCs Neotx-mLN or axillary and brachial LN (Neotx-pLN) were transplanted into these regions.

Immunization. Purified CT (Sigma-Aldrich, Taufkirchen, Germany) was administered as described previously with some modifications: ${ }^{16}$ eight weeks after transplantation mice with Neotx mLN or pLN were immunized orally with $10 \mu \mathrm{g}$ CT (in $50 \mu \mathrm{l}$ of $0.01 \mathrm{M}$ phosphatebuffered saline containing $0.2 \%$ gelatine) on days 0,8 , and 14 . On day 19 , the mice were exsanguinated, and cell suspensions were made and analyzed via flow cytometry as described below $(n=3-8)$.

Adoptive transfer. Cell suspensions of the spleen from OT-II Ly5.1 transgenic mice expressing TCR recognizing Ova were made; cells were labeled with 5-(and 6)-Carboxyfluorescein diacetate succinimidyl ester (Invitrogen, Karlsruhe, Germany) and injected intravenously into Neotx mice. Mice received a single dose of $100 \mathrm{mg}$ Ova Grade III (Sigma-Aldrich) by gavage and $10 \mathrm{mg}$ Ova by injection depending on the transplanted region (mesentery and popliteal fossa), respectively $(n=4-7)$.

Antibodies for flow cytometry. After isolation, the purity of the SCs was analyzed using anti-CD45-PE-Cy7 (BD Biosciences, Heidelberg, Germany), anti-Podoplanin-FITC (gp38; Biozol, Eching, Germany), or anti-CD31-APC (Biolegend, San Diego, CA). FDCs were detected using biotinylated anti-FDC-M2 (ImmunoKontact, Oxon, UK) and revealed by PerCP.

Cell suspensions from Neotx-mLN and Neotx-pLN $(n=3-8)$ were made and $\sim 5 \times 10^{5}$ cells were incubated with anti-CD8-PE-Cy7, antiIgA-PE (both Serotec, Oxford, UK), anti-CD4-APC, anti-CD19-APC$\mathrm{H} 7$, anti-CD11c-APC, anti-CD45-PE-Cy7, anti-CD21/35-APC (all acquired from BD Biosciences), anti-Podoplanin-PE (Biozol), and anti-Lyve-1 (kindly provided by R. Förster, Institute of Immunology, Hannover Medical School, Germany), which was shown by using a goat anti-rabbit antibody coupled with Cy3 (Dianova, Hamburg, Germany).

In three independent experiments $(n=7-8)$, OT-II cells were identified by anti-CD45.1-PE-Cy7 (BD Biosciences) and the induction of homing molecules was performed using anti-CCR9-PE (Biolegend) and anti- $\alpha_{4} \beta_{7}$ integrin-PE (BD Biosciences). All FACS analyses were performed on a FACSCanto (BD Biosciences). Isotype-matched monoclonal antibodies served as controls.
Quantification of mRNA expression. Total RNA of Neotx-mLN and Neotx-pLN ( $n=3-5)$ was isolated according to the manufacturer's protocol (ZyGEM, Hamilton, New Zealand) and cDNA synthesis was performed with $50 \mu \mathrm{M}$ oligo primer, $0.1 \mathrm{~m}$ dithiothreitol, $5 \times$ first

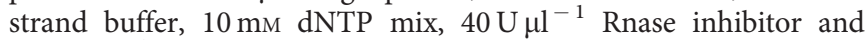

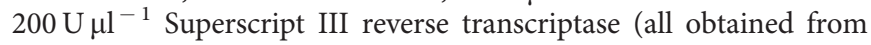
Invitrogen) in a total volume of $20 \mu \mathrm{l}$ at $50{ }^{\circ} \mathrm{C}$ for $50 \mathrm{~min}$. With this cDNA quantitative real-time PCR was performed using the QuantiTect SYBR-Green protocol from Qiagen. The primer sequences and amplicon sizes of RALDH2 (5'-GACTTGTAGCAGCTGTCTT CACT- $3^{\prime}$ and $5^{\prime}$-TCACCCATTTCTCTCCCATTTCC- $3^{\prime}$ ), IL-18 (5'-GAGGAAATGGATCCACCTGA- $3^{\prime}$ and $5^{\prime}$-ACAAACCCT CCCCACCTAAC-3'), CXCL14 (5'-TACCCACACTGCGAGGA GAAGA- $3^{\prime}$ and $5^{\prime}$-CGCTTCTCGTTCCAGGCATTGT- $3^{\prime}$ ), LTa (5'-TTCTTTGGAGCCTTTGCACT- $3^{\prime}$ and $5^{\prime}$-ACCCTTGAAACAA CGGTCAG- $3^{\prime}$ ), LTbR (5'-CCAGCACCTTCTTTCTGAGG- $3^{\prime}$ and $5^{\prime}$-GCTAGTGTCCGGTTCTGCTC-3'), CXCL13 (5'-CATAGATCG GATTCAAGTTACGCC-3' and $5^{\prime}$-GTAACCATTTGGCACGAG GATTC- $\left.3^{\prime}\right)$ and $\beta$-actin ( $5^{\prime}$-AGCCATGTACGTAGCCATCC- $3^{\prime}$ and $5^{\prime}$-CTCTCAGCTGTGGTGGTGAA-3') for the housekeeping gene were used.

Immunohistochemistry. Cryostat sections of Neotx-mLN as well as Neotx-pLN $(n=4-5)$ were fixed in acetone/methanol solution (1:1, $\left.10 \mathrm{~min},-20^{\circ} \mathrm{C}\right)$. Immunofluorescence histochemistry was performed according to standard protocols. Briefly, sections were rehydrated in TBST (0.1 м Tris, $\mathrm{pH} 7.5,0.15 \mathrm{M} \mathrm{NaCl}, 0.1 \%$ Tween-20), pre-incubated with TBST containing 5\% swine serum (Dako, Hamburg, Germany), and stained with anti CD3-APC, CD11c-APC, B220, LT $\beta R$, CD11b, (all BD Biosciences), CXCL13 (R\&D Systems, Minneapolis, MN), CCL21 (Serotec, Oxford, UK), ERTR-7 (BMA, Augst, Switzerland),FDC-M2 (ImmunoKontact, Frankfurt, Germany), CD45-APC (Caltag, Buckingham, UK), CD31-APC (Biolegend), Dlk-1 (Abcam, Cambridge, UK), gp38-Cy5, MAdCAM-1, and Lyve-1 (all kindly provided by R. Förster) in 2.5\% serum/TBST. Proliferation was visualized using anti-Ki67 (Epitomice, Burlingame, CA). Unconjugated antibodies were visualized in a second step by a goat anti-rat Cy3 (Invitrogen). To increase the EGFP signal of the transplanted cells in the regeneration experiments, an anti-GFP antibody was used and visualized by an anti-rabbit Alexa Fluor 488 (both kindly provided by C. Mühlfeld). Nuclei were visualized with DAPI staining $\left(1 \mu \mathrm{g} \mathrm{ml}^{-1} \mathrm{DAPI} / \mathrm{TBST}\right)$, and sections were mounted with Fluorescent Mounting Medium (Dako). Images were acquired using an Axiovert 200M microscope, an AxioCam MRm camera, objectives with $\times 5, \times 40$, and $\times 100$ magnification and pictures were acquired with AxioVision 4.8 software (all Carl Zeiss, Jena, Germany).

Protein multiplex analysis for the quantification of cytokines. The cytokine levels of IL-18, CXCL14, and IL-6 in the supernatants of short time SC cultures $(24 \mathrm{~h})$ were measured using Bio-Plex Pro cytokine multiplex assays (Bio-Rad, Hercules, CA). The assay was performed according to the manufacturer's instructions. In brief, $50 \mu \mathrm{l}$ supernatant was applied to color-coded beads covered with capture antibodies for IL-18, CXCL14, or IL-6. After incubation and washing, bead-bound cytokines were quantified by the biotinylated detection antibodies followed by streptavidin-PE staining. Concentrations were measured by parallel standard curves for each parameter.

Data analysis. Calculations, statistical analysis, and graphs were performed with the software Graphpad Prism 4.0 (Graphpad Software, San Diego, CA). Statistical differences were calculated in the unpaired $t$-test and are indicated by ${ }^{\star} P<0.05 ;{ }^{* *} P<0.01 ;{ }^{* * *} P<0.001$.

SUPPLEMENTARY MATERIAL is linked to the online version of the paper at http://www.nature.com/mi 


\section{ACKNOWLEDGMENTS}

We wish to thank Sheila Fryk for correction of the English. We would like to acknowledge the assistance of the Cell Sorting Core Facility of the Hannover Medical School funded in part by the Braukmann-WittenbergHerz-Stiftung and the Deutsche Forschungsgemeinschaft. The work was supported by the German Research Foundation (SFB621/A10), by the IFB-Tx, the German Federal Ministry of Education and Research, reference number: 01EO0802, and the TRR77 project A3 (to CF), and the Helmholtz Alliance Immunotherapy of Cancer.

\section{DISCLOSURE}

The authors declare no conflict of interest.

(c) 2015 Society for Mucosal Immunology

\section{REFERENCES}

1. Bajenoff, M. et al. Stromal cell networks regulate lymphocyte entry, migration, and territoriality in lymph nodes. Immunity 25, 989-1001 (2006).

2. Katakai, T., Hara, T., Lee, J.H., Gonda, H., Sugai, M. \& Shimizu, A. A novel reticular stromal structure in lymph node cortex: an immuno-platform for interactions among dendritic cells, T cells and B cells. Int. Immunol. 16, 1133-1142 (2004).

3. Bode, U. et al. Dendritic cell subsets in lymph nodes are characterized by the specific draining area and influence the phenotype and fate of primed $T$ cells. Immunology 123, 480-490 (2008).

4. Iwata, M., Hirakiyama, A., Eshima, Y., Kagechika, H., Kato, C. \& Song, S.Y. Retinoic acid imprints gut-homing specificity on Tcells. Immunity 21, $527-$ 538 (2004)

5. Bode, U., Sparmann, G. \& Westermann, J. Gut-derived effector T cells circulating in the blood of the rat: preferential re-distribution by TGF beta- 1 and IL-4 maintained proliferation. Eur. J. Immunol. 31, 2116-2125 (2001).

6. Elgueta, R. et al. Imprinting of CCR9 on CD4 T cells requires IL-4 signaling on mesenteric lymph node dendritic cells. J. Immunol. 180, 6501-6507 (2008).

7. Coombes, J.L. \& Powrie, F. Dendritic cells in intestinal immune regulation. Nat. Rev. Immunol. 8, 435-446 (2008).

8. Pabst, O. et al. Chemokine receptor CCR9 contributes to the localization of plasma cells to the small intestine. J. Exp. Med. 199, 411-416 (2004).

9. Campbell, D.J. \& Butcher, E.C. Rapid acquisition of tissue-specific homing phenotypes by CD4 $(+)$ T cells activated in cutaneous or mucosal Imphoid tissues. J. Exp. Med. 195, 135-141 (2002).

10. Snapper, C.M., Marcu, K.B. \& Zelazowski, P. The immunoglobulin class switch: beyond "accessibility". Immunity 6, 217-223 (1997).

11. Mora, J.R. et al. Generation of gut-homing IgA-secreting B cells by intestinal dendritic cells. Science 314, 1157-1160 (2006).

12. Berlin, C. et al. Alpha 4 beta 7 integrin mediates lymphocyte binding to the mucosal vascular addressin MAdCAM-1. Cell 74, 185-195 (1993).

13. Fletcher, A.L. et al. Reproducible isolation of lymph node stromal cells reveals site-dependent differences in fibroblastic reticular cells. Front. Immunol. 2, 35 (2011).

14. Hammerschmidt, S.l. et al. Stromal mesenteric lymph node cells are essential for the generation of gut-homing Tcells in vivo. J. Exp. Med. 205, 2483-2490 (2008).

15. Malhotra, D. et al. Transcriptional profiling of stroma from inflamed and resting lymph nodes defines immunological hallmarks. Nat. Immunol. 13 , 499-510 (2012).

16. Ahrendt, M., Hammerschmidt, S.I., Pabst, O., Pabst, R. \& Bode, U. Stromal cells confer lymph node-specific properties by shaping a unique microenvironment influencing local immune responses. J. Immunol. 181, 1898-1907 (2008).
17. Mebius, R.E., Breve, J., Kraal, G. \& Streeter, P.R. Developmental regulation of vascular addressin expression - a possible role for site-associated environments. Int. Immunol. 5, 443-449 (1993).

18. Wolvers, D.A.W. et al. Intranasally induced immunological tolerance is determined by characteristics of the draining lymph nodes: Studies with OVA and human cartilage gp-39. J. Immunol. 162, 1994-1998 (1999).

19. Cupedo, T., Jansen, W., Kraal, G. \& Mebius, R.E. Induction of secondary and tertiary lymphoid structures in the skin. Immunity 21, 655-667 (2004).

20. Suematsu, S. \& Watanabe, T. Generation of a synthetic lymphoid tissuelike organoid in mice. Nat. Biotechnol. 22, 1539-1545 (2004).

21. Okamoto, N., Chihara, R., Shimizu, C., Nishimoto, S. \& Watanabe, T. Artificial lymph nodes induce potent secondary immune responses in naive and immunodeficient mice. J. Clin. Invest. 117, 997-1007 (2007).

22. Muller, G., Hopken, U.E. \& Lipp, M. The impact of CCR7 and CXCR5 on lymphoid organ development and systemic immunity. Immunol. Rev. 195, 117-135 (2003).

23. Islam, S.A. et al. Mouse CCL8, a CCR8 agonist, promotes atopic dermatitis by recruiting IL-5 + T(H)2 cells. Nat. Immunol. 12, 167-177 (2011).

24. Meuter, S. et al. Murine CXCL14 is dispensable for dendritic cell function and localization within peripheral tissues. Mol. Cell Biol. 27, 983-992 (2007)

25. Gracie, J.A., Robertson, S.E. \& Mclnnes, I.B. Interleukin-18. J. Leukoc. Biol. 73, 213-224 (2003).

26. Wang, Y. \& Sul, H.S. Pref-1 regulates mesenchymal cell commitment and differentiation through Sox9. Cell Metab. 9, 287-302 (2009).

27. Benezech, C. et al. Lymphotoxin-beta receptor signaling through NF-kappaB2-RelB pathway reprograms adipocyte precursors as lymph node stromal cells. Immunity 37, 721-734 (2012).

28. Onder, L. et al. Endothelial cell-specific lymphotoxin-beta receptor signaling is critical for lymph node and high endothelial venule formation. J. Exp. Med. 210, 465-473 (2013).

29. Johansson-Lindbom, B. et al. Functional specialization of gut CD103(+) dendritic cells in the regulation of tissue-selective T cell homing. J. Exp. Med. 202, 1063-1073 (2005).

30. Khan, O., Headley, M., Gerard, A., Wei, W., Liu, L. \& Krummel, M.F. Regulation of T cell priming by lymphoid stroma. PLOS ONE 6, e26138 (2011).

31. Link, A. et al. Fibroblastic reticular cells in lymph nodes regulate the homeostasis of naive T cells. Nat. Immunol. 8, 1255-1265 (2007).

32. Forster, R., valos-Misslitz, A.C. \& Rot, A. CCR7 and its ligands: balancing immunity and tolerance. Nat. Rev. Immunol. 8, 362-371 (2008).

33. Worbs, T. et al. Oral tolerance originates in the intestinal immune system and relies on antigen carriage by dendritic cells. J. Exp. Med. 203, 519-527 (2006).

34. Luther, S.A., Tang, H.L., Hyman, P.L., Farr, A.G. \& Cyster, J.G. Coexpression of the chemokines ELC and SLC by T zone stromal cells and deletion of the ELC gene in the plt/plt mouse. Proc. Natl Acad. Sci. USA 97, 12694-12699 (2000).

35. Diehl, S. \& Rincon, M. The two faces of IL-6 on Th1/Th2 differentiation. Mol. Immunol. 39, 531-536 (2002)

36. Buettner, M., Pabst, Reinhard \& Bode, U. Lymph node stromal cells strongly influence immune response suppression. Eur. J. Immunol. 41, 624-633 (2011).

37. Onder, L. et al. IL-7-producing stromal cells are critical for lymph node remodeling. Blood 120, 4675-4683 (2012).

38. Scandella, E. et al. Restoration of lymphoid organ integrity through the interaction of lymphoid tissue-inducer cells with stroma of the T cell zone. Nat. Immunol. 9, 667-675 (2008).

39. Peduto, L. et al. Inflammation recapitulates the ontogeny of lymphoid stromal cells. J. Immunol. 182, 5789-5799 (2009). 